\title{
Preoperative Carbohydrate Load and Intraoperative Goal-Directed Fluid Therapy for Elderly Patients Undergoing Open Gastrointestinal Operation: A Prospective Randomized Controlled Trial
}

\section{Xia Liu}

Department of anesthesiology

\section{Peng Zhang}

Department of Anesthesiology

MengXue Liu

Department of Anesthesiology

JunLi Ma

Department of Anesthesiology

\section{Xinchuan Wei}

Department of Anesthesiology

Dan Fan ( $\nabla$ fandan6688@163.com )

Department of Anesthesiology

\section{Research article}

Keywords: Carbohydrate, goal-directed fluid therapy, elderly, gastrointestinal surgery, outcome

Posted Date: November 24th, 2020

DOI: https://doi.org/10.21203/rs.3.rs-112963/v1

License: (c) (i) This work is licensed under a Creative Commons Attribution 4.0 International License.

Read Full License 


\section{Abstract}

Background: At present it remains uncertain as to whether carbohydrate $(200 \mathrm{ml})$ loaded 2 hours before anesthesia induction combined with intraoperative goal-directed fluid therapy (GDFT) is beneficial to elderly patients undergoing gastrointestinal operations. As such, a randomized controlled trial was designed to evaluate the relative impact of perioperative fluid optimisation versus conventional fluid therapy (CFT) on clinical outcomes in elderly patients following gastrointestinal surgery.

Methods: A total of 120 elderly patients undergoing gastrointestinal surgery were randomized into a CFT group $(n=60)$ with traditional methods of fasting and water-deprivation, and a GDFT group $(n=60)$ with carbohydrate $(200 \mathrm{ml})$ load 2 hours before anesthesia induction. The CFT group underwent routine monitoring during surgery, however, the GDFT group was conducted by a Vigileo/FloTrac monitor with cardiac index (Cl), stroke volume variation (SVV), and mean arterial pressure (MAP). For all patients, demographic information, intraoperative parameters and postoperative outcomes were recorded.

Results: Patients in the GDFT group were administered less crystalloid fluid $(1111 \pm 442.9 \mathrm{ml}$ vs $1411 \pm$ $412.6 \mathrm{ml} ; p=0.000$ ) and produced less urine output (200 ml [150-300] vs $400 \mathrm{ml}$ [290-500]; $p=0.000$ ) relative to patients in the CFT group. Moreover, GDFT was associated with a shorter average time to first flatus ( $55 \pm 13.9$ hours vs $65 \pm 22.6$ hours; $p=0.004$ ) and oral intake ( $72 \pm 17.4$ hours vs $85 \pm 27.5$ hours; $p=0.002)$, as well as a reduction in the rate of postoperative complications ( $14(25.5 \%)$ vs $27(47.4 \%)$ patients; $p=0.016)$. However, postoperative hospitalization or hospitalization expenses were similar between groups.

Conclusions: Focused on elderly patients undergoing open gastrointestinal surgery, we found perioperative fluid optimisation may be associated with improvement of bowel function and a lower incidence of postoperative complications.

Trial registration: ChiCTR, ChiCTR1800018227. Registered 6 September 2018 - Retrospectively registered, http://www.chictr.org.cn/showproj.aspx?proj=29899

\section{Background}

Intraoperative appropriate capacity is essential in the field of anesthetic management and is of particular concern in the treatment of older adults [1]. Individuals $\geq 65$ years old are termed elderly [2]. Elderly patients typically present with a range of physical, pharmacological, and psychological comorbidities that must be carefully considered by clinicians and anesthesiologists prior to any major surgical operation [3]. Indeed, more advanced age remains a key risk factor associated with higher rates of postoperative morbidity and mortality [4].

Elderly patients scheduled to undergo gastrointestinal surgery are more likely to present to the operating room in a dehydrated or hypovolemic state as a consequence of having followed the necessary fasting and bowel preparation procedures. Intraoperative hypovolemia can, in turn, result in a range of serious 
postoperative complications including hypotension and severe arrhythmia, whereas hypervolemia can also cause serious problems such as anastomotic leaks, infection, pulmonary edema, or even death [59]. As such, it is clear that both insufficient and excess fluid infusion can cause harm in patients [10]. Owing to age-related declines in organ function and greater difficulty adjusting fluid preloading, elderly patients are thus at a substantially higher risk of postoperative mortality. It is essential that caution be taken when infusing fluids into elderly patients.

Preoperative fluid therapy mainly aims to present the patients from a dehydrated or hypovolemic state before anesthesia induction. Therefore, multiple ERAS (Enhanced Recovery After Surgery) guidelines include the oral intake of carbohydrate load $(200 \mathrm{ml}) 2$ hours before surgery, which may help to decrease the postoperative complications, such as postoperative nausea and vomiting, wound infection. Besides, the use of routine hemodynamic measurements (such as arterial blood pressure or heart rate) is often a relatively imprecise means of monitoring for changes in blood volume [11, 12]. In contrast, goal-directed fluid therapy approaches rely upon monitoring more advanced hemodynamic variables such as SVV and pulse pressure variability, which are more sensitive to hypovolemia and therefore allow for optimal preloading by enabling clinicians to appropriate titrate fluids and inotropic substances [13, 14]. A number of GDFT devices have been employed in prior trials. The Vigileo/FloTrac system commonly utilized owing to its performance being similar to that of the PiCCO system when predicting fluid responsiveness based upon [15]. In addition, this system can improve cardiovascular function and maintain an adequate intraoperative oxygen supply [16]. This procedure is also minimally invasive and simple to use, and as such this advanced hemodynamic monitor is recommended for measuring fluid responsiveness in patients undergoing high-risk major surgery [17].

GDFT has been shown to reduce the duration of hospitalization and to decrease the incidence of postoperative complications by $20-50 \%$ in previous systematic reviews [18-20]. However, there have been few studies regarding whether preoperative carbohydrate load combined with intraoperative GDFT is similarly beneficial in elderly patient populations. As such, in the present study we conducted fluid optimisation in an elderly patient population via the use of hemodynamic indicators (Cl, SVV, MAP) and vasoactive drugs, as necessary to. We hypothesized that preoperative carbohydrate $(200 \mathrm{ml})$ load and intraoperative GDFT based upon SVV and $\mathrm{Cl}$ as determined by analyzing the radial artery pulse waveform and MAP may lower postoperative hospitalization and postoperative complications in elderly patients undergoing gastrointestinal surgery.

\section{Methods}

\section{Patients}

This was a prospective study, with patients having been recruited from the Sichuan Provincial People's Hospital (Chengdu, China) between May 2018 and November 2019. The clinical trial registration number for this trial was ChiCTR1800018227, and the Ethics Committees of the Sichuan Academy of Medical Sciences and Sichuan Provincial People's Hospital approved this study. Eligible patients were individuals 
$>65$ years old that were scheduled to undergo elective open gastrointestinal surgery (anticipated operating time $>2 \mathrm{~h}$ ) and that matched the ASA (American Society of Anesthesiologists) class 2-4 criteria. Prior studies have shown that the accuracy of Vigileo/FloTrac is reduced in patients with abnormal sinus rhythm or intraabdominal pressure values [21], and as such patients were excluded from this study if they exhibited severe arrhythmia, or if they had a history of gastrointestinal surgery, peripheral artery disease, or with high risk of reflux and aspiration (pyloric obstruction or achalasia of the cardia). All patients provided written informed consent upon recruitment.

\section{Randomization and blinding}

The randomization of patients was conducted by using a series of computer-generated random numbers. The blinding of assignment for study group or control group was ensured by the opaque envelopes. However, only the patients were blind to group assignment because the attending anesthesiologist may regulate the speed of fluid infusion based on the parameters of the cardiac index trending monitor.

\section{Intraoperative management}

All patients were monitored for pulse oximetry, five-lead-electrocardiogram, blood pressure, heart rate, temperature and end-tidal carbon dioxide concentrations. Central venous pressure (CVP) monitoring was conducted in some patients as decided by the attending anesthesiologist. Patients in both groups received a minimum of one peripheral intravenous access $(18 \mathrm{G})$, with a central venous catheter being inserted via the internal jugular vein and with radial arterial catheters being introduced via the nondominant forearm under low-dose dexmedetomidine for sedation in the operating room at 7:30 AM on the day of surgery. For each patient, the following was recorded: baseline characteristics, surgery type, and basic hemodynamics variables (blood pressure, MAP, HR). An initial arterial blood gas analysis was also conducted at this time. For patients in the GDFT group, the radial arterial lines were attached to the fourth generation Vigileo/FloTrac monitor (Edwards Life Sciences, CA, USA). After making sure that the patient was not in high risk of reflux aspiration using the technique of point-of-care gastric ultrasound, anesthesia was then induced by using propofol $\left(2-2.5 \mathrm{mg} \cdot \mathrm{kg}^{-1}\right)$ or etomidate $\left(0.2 \mathrm{mg} \cdot \mathrm{kg}^{-1}\right)$ in combination with sufentanil (15-25 ug) and cisatracurium (0.15-0.2 $\left.\mathrm{mg}^{\mathrm{kg}} \mathrm{kg}^{-1}\right)$. Anesthesia was maintained with remifentanil (0.2-0.25 ug. $\left.\mathrm{kg}^{-1} \mathrm{~min}^{-1}\right)$, dexmedetomidine $\left(0.4-0.7 \mathrm{ug} \cdot \mathrm{kg}^{-1} \mathrm{~h}^{-1}\right)$ and sevoflurane at a MAC (minimum alveolar concentration) of 1-1.3 in order to achieve a BIS (bispectral index) value of 40-60. Each patient was ventilated with a tidal volume of $8 \mathrm{ml} / \mathrm{kg}$ without PEEP (positive end-expiratory pressure) at a respiratory rate sufficient to achieve an end-tidal carbon dioxide value of $35-45 \mathrm{mmHg}$. Flow rates were set to $2-3 \mathrm{~L} / \mathrm{min}$, and $60 \%$ oxygen was administered for the operative duration.

\section{Study protocol}

The bowel preparation was performed by the surgeons' instruction 1 day before surgery. The GDFT group was fasted for 8 hours and drunk $200 \mathrm{ml}$ carbohydrate (Shuneng; Yi Chang Ren Fu Pharmaceutical Co Ltd, China) before anesthesia induction. The CFT group was fasted for 8 hours and prohibited drinking for 4 hours before surgery. The CFT group was initially infused with a balanced crystalloid solution at 
$2 \mathrm{ml} / \mathrm{kg} / \mathrm{h}$ for baseline fluid therapy and $6 \mathrm{ml} / \mathrm{kg} / \mathrm{h}$ during surgery (from incision to suturing). In addition, additional bolus (crystalloid or colloids) could be infused at the discretion of the attending anesthesiologist, or vasoactive drugs could be administered to ensure that routine monitoring variables remained within the normal range (heart rate $<100 \mathrm{bpm}, \mathrm{MAP}>65 \mathrm{mmHg}$, urine output $>0.5 \mathrm{ml} / \mathrm{kg} / \mathrm{h}$ ). Patients in the GDFT treatment group were infused based on the Vigileo/FloTrac instrument. If $\mathrm{Cl}$ was $\geq$ $2.5 \mathrm{l} / \mathrm{min} / \mathrm{m}^{2}$, MAP was $>65 \mathrm{mmHg}$, and $\mathrm{SVV}$ was $<12 \%$, the administration rate was maintained at $6 \mathrm{ml} / \mathrm{kg} / \mathrm{h}$ for the entire surgical operation. When $\mathrm{Cl}$ reached $\leq 2.5 \mathrm{I} / \mathrm{min} / \mathrm{m}^{2}$ with $\mathrm{SVV}>12 \%$, additional colloid $250 \mathrm{ml}$ boluses were administered within 10 minutes. When $\mathrm{Cl}$ was $\leq 2.5 \mathrm{l} / \mathrm{min} / \mathrm{m}^{2}$ and SVV was $<12 \%$, dobutamine was injected to achieve a Cl of $\geq 2.5 \mathrm{l} / \mathrm{min} / \mathrm{m}^{2}$. Ephedrine boluses of $3-18 \mathrm{mg}$ or norepinephrine infusions were administered when $\mathrm{Cl}$ was $\geq 2.5 \mathrm{I} / \mathrm{min} / \mathrm{m}^{2}$ and MAP was $<65 \mathrm{mmHg}$ (Fig. 2). All hemodynamic variables were reassessed after 10 minutes.

\section{Data collection}

We recorded HR and MAP values at 5 min prior to the induction of anesthesia (T1), 30 min after the operation was initiated (T2), 60 min after the operation was initiated (T3), 90 min after the operation was initiated (T4), 120 min after the operation was initiated (T5), and at the end of surgery (T6). We additionally measured arterial lactate concentrations at T1, T3, and T6. All intraoperative data were collected upon the completion of the operation, including operative duration, duration of anesthetization, types and amounts of fluid infusion, estimated blood loss, urine output, and vasoactive agent use. Following intensive care unit (ICU) or ward admission, the management of all patients was conducted by medical staff blinded to patient group assignments. Time to first flatus and time to first oral intake was recorded. Following patient discharge, we recorded the length of hospitalization as well as postoperative complications. The predefined criteria for complications in the study protocol were described in Additional 1. At first, we determined the length of hospitalization as the primary outcome and the rate of postoperative complications as secondary outcome. However, during analysis of some experimental data, we noted that the reduction in the length of hospitalization was lower than that we had been predicted in the original study design. Moreover, we found that the interventions may have more effect on the rate of postoperative complications than the length of hospitalization. Therefore, we changed the rate of postoperative complications for primary outcome. Moreover, we calculated the sample size once again based on the new primary outcome.

\section{Statistical analysis}

Sample size calculation was according to a prospective analysis of postoperative hospital stay and the rate of postoperative complications for patients undergoing major bowel surgery [22]. The rate of postoperative complication in the study was $59.3 \%$ in the control group and $37.5 \%$ in the treatment group. For a reduction in the postoperative complication from 60 to $32 \%$ with type I error of 0.05 and a power of $80 \%$, a sample size of 50 patients was calculated by our researchers for each group. Owing to $20 \%$ patients with changes in surgical procedure, our study finally included 60 patients per group. SPSS 26.0 (IBM, IL, USA) was used for all statistical testing. Data were given as mean $\pm S D$, absolute values (percentages) or medians (25th -75th percentiles). Data were compared via Student's t-tests (for normally 
distributed variables), Wilcoxon rank-sum tests (for non-normally distributed variables), and chi-squared or Fisher's exact tests (for categorical variables). Normality was assessed via the Kolmogorov-Smirnov test. Hemodynamic variables (MAP or HR) that were normally compared via repeated measures one-way ANOVAs, whereas they were compared via the Friedman test when non-normally distributed. $p<0.05$ was the significance threshold.

\section{Results}

In total, 225 patients were eligible for inclusion in the present study between May 2018 and November 2019. After excluding patients based on our exclusion criteria and failure to give consent, the remaining 120 patients were randomized into the GDFT $(n=60)$ and CFT $(n=60)$ groups. Among these patients, $5(2$ and 3 in the GDFT and CFT groups, respectively) dropped out of the study, 3 ( 1 and 2 in the GDFT and CFT groups, respectively) were found to be inoperable (Fig. 1). In total, our final analyses thus included 55 patients in the GDFT group and 57 patients in the CFT group.

There were no significant differences between groups with respect to demographic characteristics, surgical category, or ASA classification ( $p>0.05$ ). Operative duration and duration of anesthetization were also similar between groups. While intraoperative blood loss was comparable between groups, patients in the CFT group were administered a significantly larger volume of fluids relative to patients in the GDFT group ( $2033 \pm 468.7 \mathrm{ml}$ vs $1697 \pm 536.9 \mathrm{ml} ; p=0.001$ ), with the difference primarily being in the form of crystalloid infusion ( $1411 \pm 412.6 \mathrm{ml}$ vs $1111 \pm 442.9 \mathrm{ml} ; p=0.000)$. Urine out was also significantly greater in the CFT group compared with the GDFT group ( $400 \mathrm{ml}$ [290-500] vs $200 \mathrm{ml}$ [150300]; $p=0.000$ ). There were no significant differences between groups with respect to intraoperative colloid infusion volume. With respect to vasoactive drugs, dobutamine was used more frequently in the GDFT group. For full details regarding these findings, see Tables 1 and 2 . 
Table 1

Patient baseline characteristics

\begin{tabular}{|c|c|c|c|}
\hline \multicolumn{4}{|c|}{ GDFT group $(n=55)$ CFT group $(n=57) P$} \\
\hline Age (years) & $68.3 \pm 5.49$ & $69.2 \pm 6.55$ & 0.433 \\
\hline Height (m) & $1.61 \pm 0.07$ & $1.62 \pm 0.08$ & 0.353 \\
\hline Weight (kg) & $60.20 \pm 10.53$ & $59.26 \pm 10.62$ & 0.640 \\
\hline $\mathrm{BMI}\left(\mathrm{kg} / \mathrm{m}^{2}\right)$ & $23.02 \pm 2.75$ & $22.34 \pm 3.11$ & 0.223 \\
\hline $\operatorname{Sex}(M / F)$ & $36 / 19$ & $40 / 17$ & 0.593 \\
\hline ASA II/III/IV & $38 / 12 / 5$ & $40 / 10 / 7$ & 0.767 \\
\hline Type of surgery & $16(29.1 \%)$ & $15(26.3 \%)$ & 0.718 \\
\hline Gastrectomy & $20(36.4 \%)$ & $25(43.9 \%)$ & \\
\hline Colectomy & $19(34.5 \%)$ & 17(29.8\%) & \\
\hline \multicolumn{4}{|l|}{ Proctectomy } \\
\hline
\end{tabular}


Table 2

Comparison of intraoperative surgery and anesthesia

\begin{tabular}{|c|c|c|c|}
\hline Duration of anesthesia (min) & $254 \pm 64.8$ & $244 \pm 60.6$ & 0.413 \\
\hline Duration of surgery (min) & $208 \pm 59.9$ & $200 \pm 57.1$ & 0.469 \\
\hline Estimated blood loss (ml) & $200(150-300)$ & $200(100-400)$ & 0.773 \\
\hline Total intraoperative fluids (ml) & $1697 \pm 536.9$ & $2033 \pm 468.7$ & 0.001 \\
\hline Crystalloid (ml) & $1111 \pm 442.9$ & $1411 \pm 412.6$ & 0.000 \\
\hline Colloid (ml) & $623 \pm 211.9$ & $586 \pm 216.3$ & 0.370 \\
\hline Urine output (ml) & $200(150-300)$ & $400(290-500)$ & 0.000 \\
\hline Intraoperative vasoactive drugs & 25 & 31 & 0.345 \\
\hline Ephedrine & 10 & 4 & 0.091 \\
\hline Norepinephrine & 9 & 2 & 0.022 \\
\hline Dobutamine & 2 & 3 & 1.000 \\
\hline Nitroglycerin & & & \\
\hline
\end{tabular}

The hemodynamic data for patients in these two fluid management groups were additionally measured, and presented in Table 3. Relative to baseline, the MAP of patients in the GDFT group was significantly decreased at T3 $(84.8 \pm 11.2 \mathrm{mmHg}$ vs $94.5 \pm 11.9 \mathrm{mmHg} ; p=0.000), \mathrm{T} 4(83.5 \pm 10.5 \mathrm{mmHg}$ vs $94.5 \pm$ $11.9 \mathrm{mmHg} ; p=0.000)$, and T5 (86.4 $\pm 10.6 \mathrm{mmHg}$ vs $94.5 \pm 11.9 \mathrm{mmHg} ; p=0.002)$. Similarly, the HR of patients in the GDFT group also reduced at T3 (67/min (64-75) vs $74 / \mathrm{min}(67-84) ; p=0.002)$,T4 $(66 / \mathrm{min}(62-72)$ vs $74 / \mathrm{min}(67-84) ; p=0.000)$, and T5 (66/min (62-71) vs $74 / \mathrm{min}(67-84) ; p=0.000)$. In the CFT group, we also detected significant reductions in MAP at T2 $(84.0 \pm 11.0 \mathrm{mmHg}$ vs $94.1 \pm$ $11.4 \mathrm{mmHg} ; p=0.000)$, T3 $(85.0 \pm 11.3 \mathrm{mmHg}$ vs $94.1 \pm 11.4 \mathrm{mmHg} ; p=0.000), \mathrm{T} 4(83.4 \pm 9.6 \mathrm{mmHg}$ vs $94.1 \pm 11.4 \mathrm{mmHg} ; p=0.000)$, and T5 $(85.9 \pm 8.3 \mathrm{mmHg}$ vs $94.1 \pm 11.4 \mathrm{mmHg} ; p=0.000)$ and significant decreases in HR at T3 (64/min (60-70) vs $75 / \mathrm{min}(66-81) ; p=0.002)$ and T4 (67/min (62-71) vs $75 / \min (66-81) ; p=0.034)$ relative to baseline. The MAP of patients in the GDFT group was also significantly higher than that of patients in the CFT group at T2 $(89.9 \pm 10.5 \mathrm{mmHg}$ vs $84.0 \pm 11.0 \mathrm{mmHg}$; $p=0.005)$. There was also a significant increase in arterial lactate concentration in the CFT group relative to baseline at the end of surgery $(1.0 \mathrm{mmol} / \mathrm{l}(0.8-1.2) \mathrm{vs} 0.7 \mathrm{mmol} / \mathrm{l}(0.6-0.95) ; p=0.000)$. No other differences in MAP, HR, or lactate values were detected between these two groups. 
Table 3

intraoperative hemodynamic data and blood lactate concentrations

\begin{tabular}{|c|c|c|c|}
\hline \multicolumn{4}{|c|}{ GDFT group $(n=55)$ CFT group $(n=57) P$} \\
\hline MAP $(\mathrm{mmHg})$ & $94.5 \pm 11.9$ & $94.1 \pm 11.4$ & 0.862 \\
\hline $\mathrm{T} 1$ & $89.9 \pm 10.5$ & $84.0 \pm 11.0 * \star \star$ & $0.005^{\#}$ \\
\hline $\mathrm{T} 2$ & $84.8 \pm 11.2 * \star \star$ & $85.0 \pm 11.3 * \star \star$ & 0.945 \\
\hline T3 & $83.5 \pm 10.5 * \star \star$ & $83.4 \pm 9.6 * * \star$ & 0.941 \\
\hline $\mathrm{T} 4$ & $86.4 \pm 10.6$ ** & $85.9 \pm 8.3 * \star \star$ & 0.771 \\
\hline T5 & $90.6 \pm 11.6$ & 92. $2 \pm 14.0$ & 0.504 \\
\hline T6 & $74(67-84)$ & $75(66-81)$ & 0529 \\
\hline $\mathrm{HR}(/ \min )$ & $67(63-78)$ & $67(64-75)$ & 0.753 \\
\hline $\mathrm{T} 1$ & $67(64-75)$ ** & $64(60-70)$ ** & 0.208 \\
\hline $\mathrm{T} 2$ & $66(62-72) * * *$ & $67(62-71)$ * & 0.907 \\
\hline T3 & $66(62-71) * * *$ & $68(62-72)$ & 0.720 \\
\hline T4 & $69(65-77)$ & $71(66-77)$ & 0.433 \\
\hline T5 & $0.8(0.6-1.3)$ & $0.7(0.6-0.95)$ & 0.363 \\
\hline T6 & $0.9(0.6-1.3)$ & $0.8(0.65-1.1)$ & 0.243 \\
\hline Lactate $(\mathrm{mmol} / \mathrm{l})$ & $0.9(0.7-1.3)$ & $1.0(0.8-1.2) * \star *$ & 0.752 \\
\hline
\end{tabular}

T1

T3

T6

Note: Values are presented as medians (25th -75 th percentiles) or means \pm SD (standard deviation); $\mathrm{MAP}=$ mean arterial pressure; $\mathrm{HR}=$ heart rate; $* p<0.05$, $* \star p<0.01, * \star \star p<0.001$ analysis of variance by one-way ANOVA or Friedman test against basic value; ${ }^{*} p<0.05$ GDFT groups. CFT group (t-test). $\mathrm{T} 1=5 \mathrm{~min}$ prior to induction of anesthesia; $\mathrm{T} 2=30 \mathrm{~min}$ after the initiation of surgery; $\mathrm{T} 3=60 \mathrm{~min}$ after the initiation of surgery; T4 = 90 min after the initiation of surgery; T5=120 min after the initiation of surgery; $\mathrm{T} 6=$ at the end of surgery.

With respect to postoperative bowel function, we found that GDFT was associated with a shorter time to first flatus ( $55 \pm 13.9$ hours vs $65 \pm 22.6$ hours; $p=0.004$ ) and to first oral intake ( $72 \pm 17.4$ hours vs $85 \pm$ 27.5 hours; $p=0.002$ ) relative to the CFT group. In addition, there were significantly fewer instances of postoperative complications among patients in the GDFT group relative to the CFT group (14 (25.5\%) vs $27(47.4 \%)$ patients; $p=0.016)$. Further analysis of predefined types of complications found that there was a lower rate of infection complications in in the GDFT group relative to the CFT group $(5(9.1 \%)$ vs 13 $(22.8 \%)$ patients; $p=0.048)$. However, GDFT was not associated with any significant differences in 
postoperative hospitalization duration, ICU admission, or hospitalization expenses. Although there were no significant differences between groups with respect to postoperative mortality, two patients in the CFT group did die on postoperative day 3 as a result of acute massive gastrointestinal hemorrhage and acute myocardial infraction respectively. For further details, see Table 4.

Table 4

Comparison of postoperative outcomes and complications

\begin{tabular}{|llll|}
\hline & $\begin{array}{l}\text { GDFT group } \\
(\mathbf{n = 5 5 )}\end{array}$ & $\begin{array}{l}\text { CFT group } \\
(\mathbf{n}=\mathbf{5 7})\end{array}$ & $\boldsymbol{P}$ \\
\hline Exhaust time (hours) & $55 \pm 13.9$ & $65 \pm 22.6$ & 0.004 \\
\hline Oral taken (hours) & $72 \pm 17.4$ & $85 \pm 27.5$ & 0.002 \\
\hline Postoperative hospitalization (days) & $9.2 \pm 2.9$ & $9.7 \pm 3.1$ & 0.397 \\
\hline Amount hospital charges (RMB) & $56845 \pm 16446$ & $60571 \pm 16579$ & 0.235 \\
\hline Admission to ICU & $5(9.1 \%)$ & $6(10.5 \%)$ & 0.799 \\
\hline Postoperative mortality & $0(0 \%)$ & $2(3.5 \%)$ & 0.496 \\
\hline Patients with complications & & & 0.048 \\
\hline Infection & $5(9.1 \%)$ & $13(22.8 \%)$ & 0.284 \\
\hline Respiratory & $5(9.1 \%)$ & $9(15.8 \%)$ & 0.448 \\
\hline Cardiovascular & $6(10.9 \%)$ & $9(15.8 \%)$ & 0.316 \\
\hline Abdominal & $6(10.9 \%)$ & $10(17.5 \%)$ & 1.000 \\
\hline Renal & $1(1.8 \%)$ & $2(3.5 \%)$ & 1.000 \\
\hline Others & $2(3.6 \%)$ & $2(3.5 \%)$ & 0.016 \\
\hline Total patients with complications & $14(25.5 \%)$ & $27(47.4 \%)$ & \\
\hline $\begin{array}{l}\text { Note: Values are absolute (percentage) or mean } \pm \text { SD (standard deviation). RMB = Renminbi; ICU }= \\
\text { intensive care unit. }\end{array}$ & & \\
\hline
\end{tabular}

\section{Discussion}

In this prospective randomized study, we compared outcomes in elderly patients undergoing open gastrointestinal surgery that were treated via traditional standard of preoperative fasting and CFT or via perioperative fluid optimisation including preoperative carbohydrate load and intraoperative GDFT. This analysis revealed that the latter was associated with more rapid restoration of bowel function and with a lower rate of postoperative complications. 
For the present study, we specifically analyzed outcomes in elderly patients undergoing open gastrointestinal surgery for two reasons. First, elderly patients with gastric or colorectal cancer have high rates of comorbidities that are associated with increased surgical risk [23]. Second, unlike laparoscopic surgery, open gastrointestinal surgery is associated with large changes in intraoperative fluid levels [24], and as such poor organ perfusion or fluid overload have the potential to lead to poorer surgical outcomes. Further study of optimal care techniques for elderly patients undergoing major abdominal surgery is needed, and as such in the present study we sought to determine whether the reduction of the time for preoperative fasting and GDFT was able to improve clinical outcomes in these patients.

For this study, we opted to utilize $\mathrm{Cl}$ rather than the oxygen delivery index as the key target variable for our GDFT protocol as it can be readily and continuously monitored via radial artery pulse waveform analysis. When arterial oxygen saturation and hemoglobin levels are adequate, $\mathrm{Cl}$ can serve as an effective means of evaluating oxygen supply within tissues and organs [25]. As the use of $\mathrm{Cl}$ and SVV with the Vigileo/FloTrac monitor has been found to be potentially unreliable in patients with irregular heart rhythms [21,26] and in patients with poorly controlled intraoperative ventilation [27], we did not include patients with severe arrhythmia in the present study, and all patients were ventilated using a tidal volume of $8 \mathrm{ml} / \mathrm{kg}$.

Excess fluid transfusion can result in a range of problems including increased rates of postoperative cardiac morbidity, pneumonia, respiratory failure, delayed wound healing, and anastomotic leak as a consequence of intestinal edema in patients undergoing colorectal surgery [8]. As shown in some prior trials $[16,25,28]$, our study also found that the GDFT protocol is associated with reductions in the rate of postoperative complications. In addition, the lower incidence of postoperative infection complications in the GDFT group may also be related to preoperative oral carbohydrate loading, which may reduce the metabolic and inflammatory response after surgery and improved surgical clinical outcomes [29]. However, the rates of postoperative complications in some previous studies were higher than in our present analysis. There are two potential reasons for this discrepancy. For one, Mayer et al. [25] selected high-risk patients with at least two risk factors according to risk index of Lee [30] as their experimental subjects. Furthermore, Benes et al. [28] recruited patients that had to meet both operation-related and patient-related high-risk criteria. In contrast, all patients we included were over the age of 65 , some of whom may be in the low-to-moderate risk category. In addition, we only monitored the incidence of postoperative complications that occurred during hospitalization, whereas Benes et al. monitored patients for 30 days [28]. Together, our results suggest that perioperative fluid optimisation reduces the incidence of postoperative complications in elderly patients, rather than only in high-risk patients.

The relationship between GDFT and the duration of postoperative hospitalization remains controversial. Early studies found GDFT to be associated with a reduction in the duration of postoperative hospitalization [25], but this finding was not repeated in more recent studies [31], particularly in studies performed in an ERAS (enhanced recovery after surgery) setting [2,32]. The increased application of ERAS protocols has led to improvements in the quality of perioperative care in control groups over the past 15 years, potentially influencing these study results [10]. However, hospitalization duration can also 
be influenced by a range of other factors such as patients' wishes, preoperative physical condition, health care system requirements, and institution-specific differences in treatment regimens. All of these factors thus have the potential to influence the relationship between GDFT and postoperative hospitalization duration.

Given that it is a sensitive indicator of organ perfusion, arterial lactate levels are closely linked to tissue hypoxia and blood volume insufficiency [33]. In the context of suboptimal fluid management, postoperative restoration of gastrointestinal motility and oral food intake may be delayed due to higher lactate levels. While at baseline in the present study we found that lactate levels were higher in the GDFT group relative to the CFT group, we did not observe any significant differences between these values at analyzed time points. However, we did find that lactate values in the CFT group increased at the end of surgery relative to baseline values. The slower recovery of bowel function observed in the CFT group may thus be associated with increased lactate production.

Urine output is typically utilized to approximate renal function and blood volume. In this study, we found that patients in the GDFT treatment group required reduced crystalloid administration and exhibited reduced urine output relative to patients in the CFT group. Postoperative renal failure occurred in one patient in the GDFT group and two patients in the CFT group. This outcome may be explained by work conducted by Kheterpal et al [34], as these authors suggested that urine is unreliable when used as a marker of blood volume and renal function, given that it can be influenced by neurohormonal signaling in response to operative stress. The administration of diuretics and vasoactive agents has also been linked to the incidence of acute renal failure.

There are multiple limitations to the present analysis. For one, in an effort to avoid potential compounds we did not utilize the $\mathrm{Cl}$ and SVV trending monitor for patients in the CFT group, so we were not able to compare these parameters between groups. Moreover, we were not able to blind investigators intraoperatively to patient treatment strategies. Additionally, intraoperative attending anesthesiologists were the primary focus of this study. The professional level of the attending anesthesiologists and physicians for postoperative treatment in the ICU and hospital wards should be equivalent between groups. Otherwise, it is possible that higher rates of postoperative complications may result from poorer postoperative care in the CFT group. Lastly, we did not pre-define standardized discharge criteria in the present study, as discharge may be influenced by a range of factors including patient demands, health care system capacity, and specific treatment regimens used. Thus, the duration of postoperative hospitalization could be also biased.

\section{Conclusion}

The results of this study indicate that preoperative carbohydrate load and intraoperative fluid optimization guided by $\mathrm{Cl}, \mathrm{SVV}$, and MAP may be associated with more rapid improvements in bowel function and a decreased incidence of postoperative complications in elderly patients undergoing open 
gastrointestinal surgery. However, this GDFT treatment strategy was not associated with any decreases in postoperative hospitalization.

\section{Abbreviations}

GDFT = goal-directed fluid therapy; CTF = conventional fluid therapy; $\mathrm{BMI}=$ body mass index; $\mathrm{ASA}=$ American Society of Anesthesiologists; $\mathrm{MAP}=$ mean arterial pressure; $\mathrm{HR}=$ heart rate; $\mathrm{RMB}=$ Renminbi; $\mathrm{ICU}=$ intensive care unit.

\section{Declarations}

Acknowledgements

Not applicable.

Authors' contributions

PZ, XCW and DF contributed to the concept and design of the study. XL, MXLand JLM collected and analyzed the data. $X L$ wrote the original draft. $P Z, X C W$ and DF critically reviewed and revised the manuscript. All authors have read and approved the manuscript.

Funding

This work was Funded by the Sichuan science and technology department research projects, China (No. 2017FZ0042).

Availability of data and materials

All data generated or analyzed during this study are included in this published article and supporting data can be obtained from the corresponding author.

Ethics approval and consent to participate

This study was approved by the Ethics Committees of the Sichuan Academy of Medical Sciences and Sichuan Provincial People's Hospital and written informed consent was abtained from all patients.

Consent for publication

Not Applicable.

Competing interests

The authors declare they have no competing interests.

Author details 
a North Sichuan Medical College, Nanchong, Sichuan, China. Department of anesthesiology, Sichuan Academy of Medical Sciences and Sichuan Provincial People's Hospital, Chengdu, Sichuan, China.

b North Sichuan Medical College, Nanchong, Sichuan, China.

\section{References}

1. Gustafsson U, Scott M, Schwenk W, Demartines N, Roulin D, Francis N ,et al. Guidelines for perioperative care in elective colonic surgery: Enhanced Recovery After Surgery (ERAS $(\circledR)$ ) Society recommendations. World J Surg. 2013; 37(2):259-284.

2. Lohsiriwat V. Outcome of Enhanced Recovery After Surgery (ERAS) for Colorectal Surgery in Early Elderly and Late Elderly Patients. Ann Acad Med Singapore. 2019; 48:347-353.

3. Evers BM, Townsend CM, Thompson JC. Organ Physiology of Aging. Surg Clin North Am. 1994; 74(1):23-39.

4. Turrentine FE, Wang H, Simpson VB, Jones RS. Surgical Risk Factors, Morbidity, and Mortality in Elderly Patients. Journal of the American College of Surgeons. 2006; 203(6):865-877.

5. Nisanevich V, Felsenstein I, Almogy G, Weissman C, Einav S, Matot I. Effect of Intraoperative Fluid Management on Outcome after Intraabdominal Surgery. Anesthesiology. 2005; 103(1):25-32.

6. Lobo DN, Bostock KA, Neal KR, Perkins AC, Rowlands BJ, Allison SP. Effect of salt and water balance on recovery of gastrointestinal function after elective colonic resection: a randomised controlled trial. The Lancet. 2002; 359(9320):1812-1818.

7. Brandstrup B, Tønnesen H, Beier-Holgersen R, Hjorts $\varnothing$ E, Ørding H, Lindorff-Larsen $K$, et al. Effects of Intravenous Fluid Restriction on Postoperative Complications: Comparison of Two Perioperative Fluid Regimens. Annals of Surgery. 2003; 238(5):641-648.

8. Holte K, Sharrock NE, Kehlet H. Pathophysiology and clinical implications of perioperative fluid excess. Br J Anaesth. 2002; 89(4):622-632.

9. Strunden MS, Heckel K, Goetz AE, Reuter DA. Perioperative fluid and volume management: physiological basis, tools and strategies. Ann Intensive Care. 2011; 1(1).

10. Miller TE, Roche AM, Mythen M. Fluid management and goal-directed therapy as an adjunct to Enhanced Recovery After Surgery (ERAS). Canadian Journal of Anesthesia/Journal canadien d'anesthésie. 2014; 62(2):158-168.

11. Pierrakos C, Velissaris D, Scolletta S, Heenen S, De Backer D, Vincent J-L. Can changes in arterial pressure be used to detect changes in cardiac index during fluid challenge in patients with septic shock? Intensive Care Medicine. 2012; 38(3):422-428.

12. Meregalli A, Oliveira RP, Friedman G. Occult hypoperfusion is associated with increased mortality in hemodynamically stable, high-risk, surgical patients. Crit Care. 2004; 8(2):R60.

13. Tote SP, Grounds RM. Performing perioperative optimization of the high-risk surgical patient. $\mathrm{Br} \mathrm{J}$ Anaesth. 2006; 97(1):4-11. 
14. Davies SJ, Wilson RJT. Preoperative optimization of the high-risk surgical patient. $\mathrm{Br} \mathrm{J}$ Anaesth. 2004; 93(1):121-128.

15. Hofer CK, Senn A, Weibel L, Zollinger A. Assessment of stroke volume variation for prediction of fluid responsiveness using the modified FloTrac ${ }^{\text {TM }}$ and PiCCOplus ${ }^{\text {TM }}$ system. Crit Care. 2008; 12(3):R82.

16. Salzwedel C, Puig J, Carstens A, Bein B, Molnar Z, Kiss K ,et al. Perioperative goal-directed hemodynamic therapy based on radial arterial pulse pressure variation and continuous cardiac index trending reduces postoperative complications after major abdominal surgery: a multi-center, prospective, randomized study. Crit Care. 2013; 17(5):R191.

17. Miller T, Myles P. Perioperative Fluid Therapy for Major Surgery. Anesthesiology. 2020; 132(2):405405.

18. Pearse RM, Harrison DA, MacDonald N, Gillies MA, Blunt M, Ackland G ,et al. Effect of a Perioperative, Cardiac Output-Guided Hemodynamic Therapy Algorithm on Outcomes Following Major Gastrointestinal Surgery. JAMA. 2014; 311(21):2181.

19. Gurgel ST, do Nascimento P. Maintaining Tissue Perfusion in High-Risk Surgical Patients. Anesth Analg. 2011; 112(6):1384-1391.

20. Hamilton MA, Cecconi M, Rhodes A. A Systematic Review and Meta-Analysis on the Use of Preemptive Hemodynamic Intervention to Improve Postoperative Outcomes in Moderate and HighRisk Surgical Patients. Anesth Analg. 2011; 112(6):1392-1402.

21. Lansdorp B, Lemson J, van Putten MJAM, de Keijzer A, van der Hoeven JG, Pickkers P. Dynamic indices do not predict volume responsiveness in routine clinical practice. $\mathrm{Br} \mathrm{J}$ Anaesth. 2012; 108(3):395-401.

22. Wakeling HG, McFall MR, Jenkins CS, Woods WGA, Miles WFA, Barclay GR ,et al. Intraoperative oesophageal Doppler guided fluid management shortens postoperative hospital stay after major bowel surgery. Br J Anaesth. 2005; 95(5):634-642.

23. Nagata T, Tamai M, Taniguchi A, Kamada Y, Fukuda K, Iwata G ,et al. Impact of Preoperative Comorbidities on Long-Term Prognosis in Elderly Patients with Gastric and Colorectal Cancer. Gan to Kagaku ryoho Cancer \& Chemotherapy. 2019; 46(13):2282-2284.

24. Sujatha PP, Nileshwar A, Krishna HM, Prasad SS, Prabhu M, Kamath SU. Goal-Directed vs Traditional Approach to Intraoperative Fluid Therapy during Open Major Bowel Surgery: Is There a Difference? Anesthesiology Research and Practice. 2019; 2019:1-11.

25. Mayer J, Boldt J, Mengistu AM, Röhm KD, Suttner S. Goal-directed intraoperative therapy based on autocalibrated arterial pressure waveform analysis reduces hospital stay in high-risk surgical patients: a randomized, controlled trial. Crit Care. 2010; 14(1):R18.

26. Umgelter A, Reindl W, Schmid RM, Huber W. Is supra-ventricular arrhythmia a reason for the bad performance of the FlowTrac device? Crit Care. 2007; 11(1):406.

27. Kubitz JC, Annecke T, Forkl S, Kemming GI, Kronas N, Goetz AE ,et al. Validation of pulse contour derived stroke volume variation during modifications of cardiac afterload. $\mathrm{Br} \mathrm{J}$ Anaesth. 2007; 98(5):591-597. 
28. Benes J, Chytra I, Altmann P, Hluchy M, Kasal E, Svitak R ,et al. Intraoperative fluid optimization using stroke volume variation in high risk surgical patients: results of prospective randomized study. Crit Care. 2010; 14(3):R118.

29. Rizvanović N, Nesek Adam V, Čaušević S, Dervišević S, Delibegović S. A randomised controlled study of preoperative oral carbohydrate loading versus fasting in patients undergoing colorectal surgery. Int J Colorectal Dis. 2019; 34(9):1551-1561.

30. Lee TH, Marcantonio ER, Mangione CM, Thomas EJ, Polanczyk CA, Cook EF ,et al. Derivation and Prospective Validation of a Simple Index for Prediction of Cardiac Risk of Major Noncardiac Surgery. Circulation. 1999; 100(10):1043-1049.

31. Cesur S, Çardaközü T, Kuş A, Türkyılmaz N, Yavuz Ö. Comparison of conventional fluid management with PVI-based goal-directed fluid management in elective colorectal surgery. J Clin Monit Comput. 2018; 33(2):249-257.

32. Rollins KE, Lobo DN. Intraoperative Goal-directed Fluid Therapy in Elective Major Abdominal Surgery. Annals of Surgery. 2016; 263(3):465-476.

33. Forget P, Lois F, de Kock M. Goal-Directed Fluid Management Based on the Pulse Oximeter-Derived Pleth Variability Index Reduces Lactate Levels and Improves Fluid Management. Anesth Analg. 2010:1.

34. Kheterpal S, Tremper Kevin K, Englesbe Michael J, O’Reilly M, Shanks Amy M, Fetterman Douglas M ,et al. Predictors of Postoperative Acute Renal Failure after Noncardiac Surgery in Patients with Previously Normal Renal Function. Anesthesiology. 2007; 107(6):892-902.

\section{Figures}




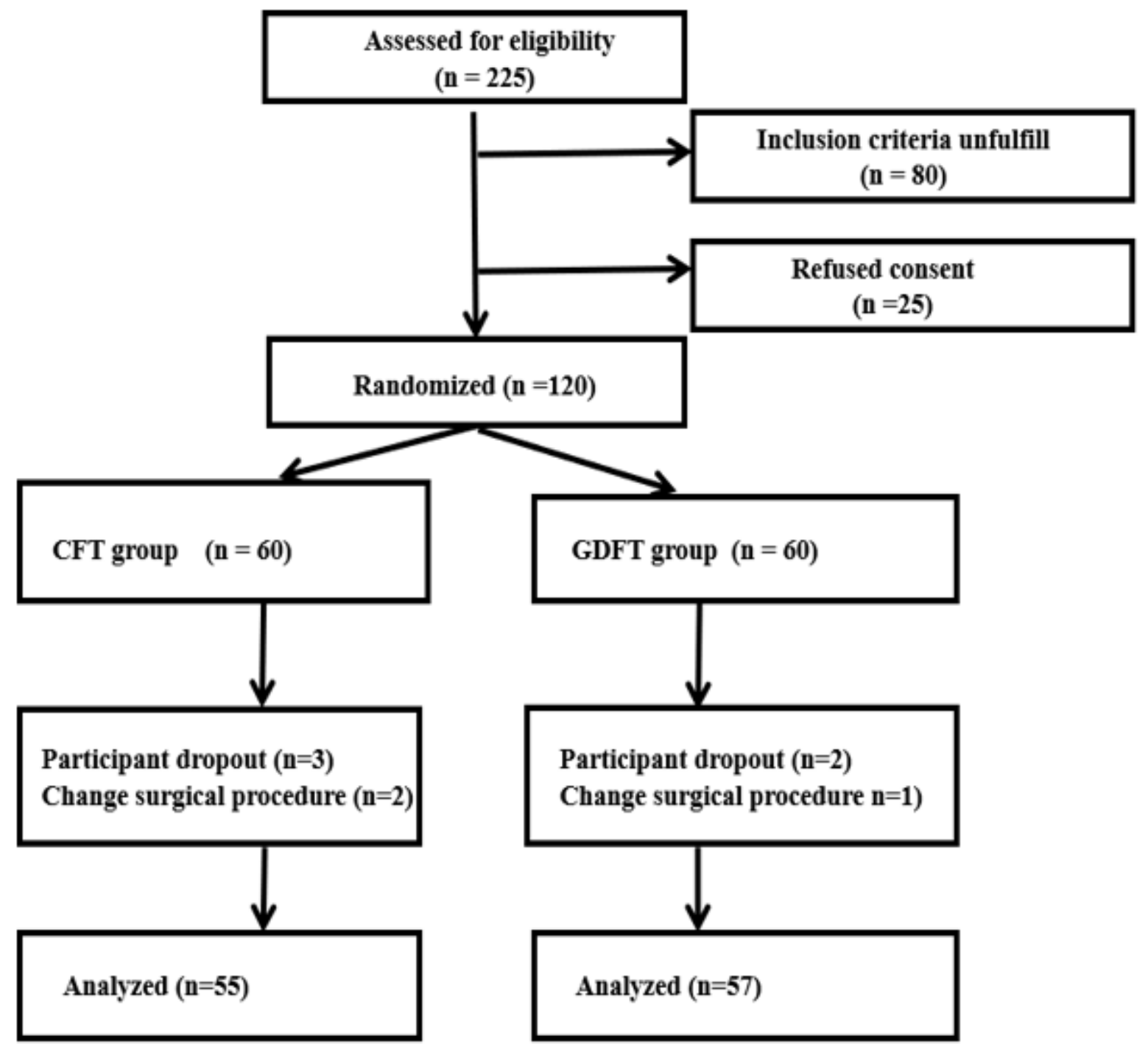

Figure 1

Trial flow chart 


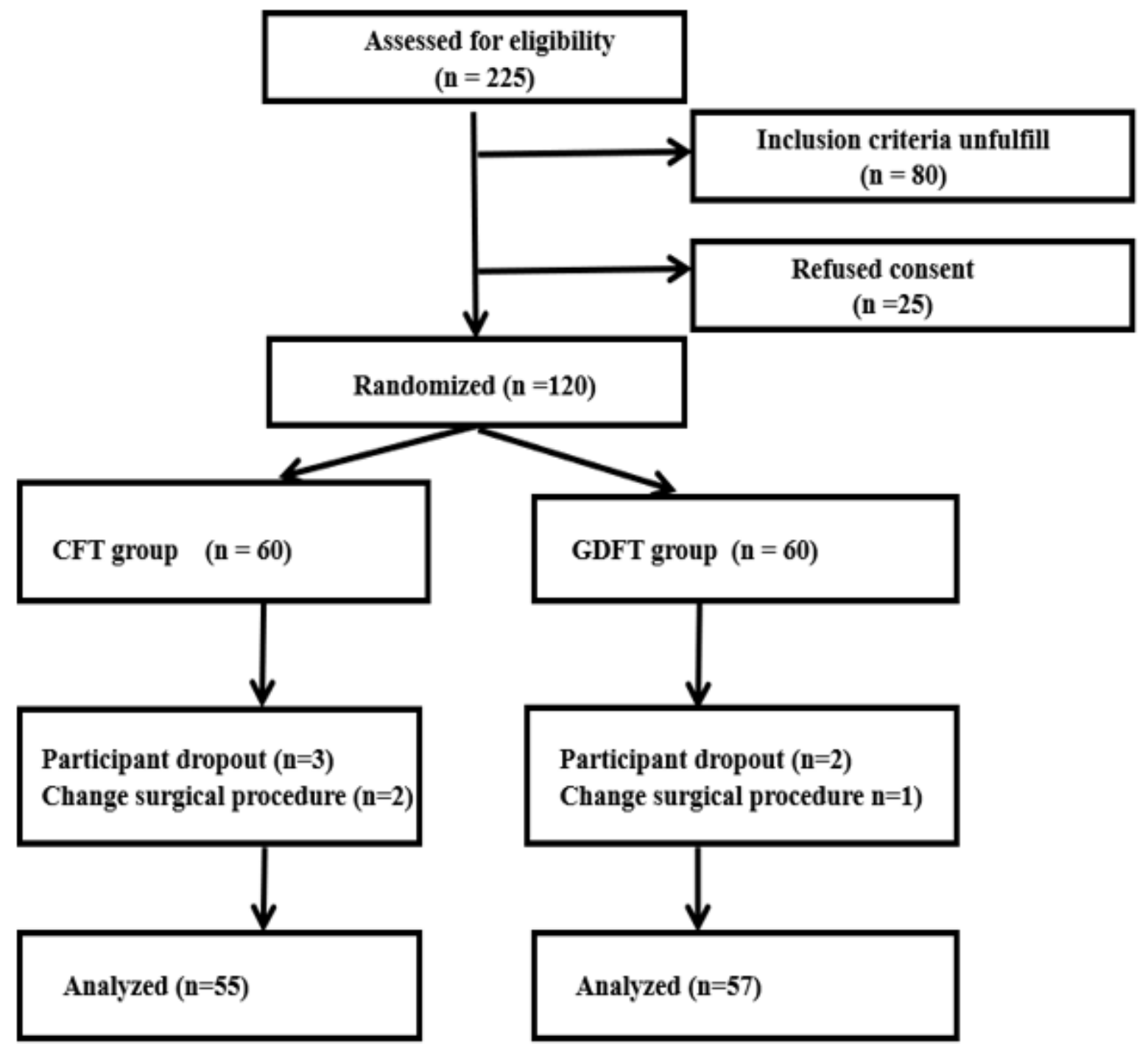

Figure 1

Trial flow chart 


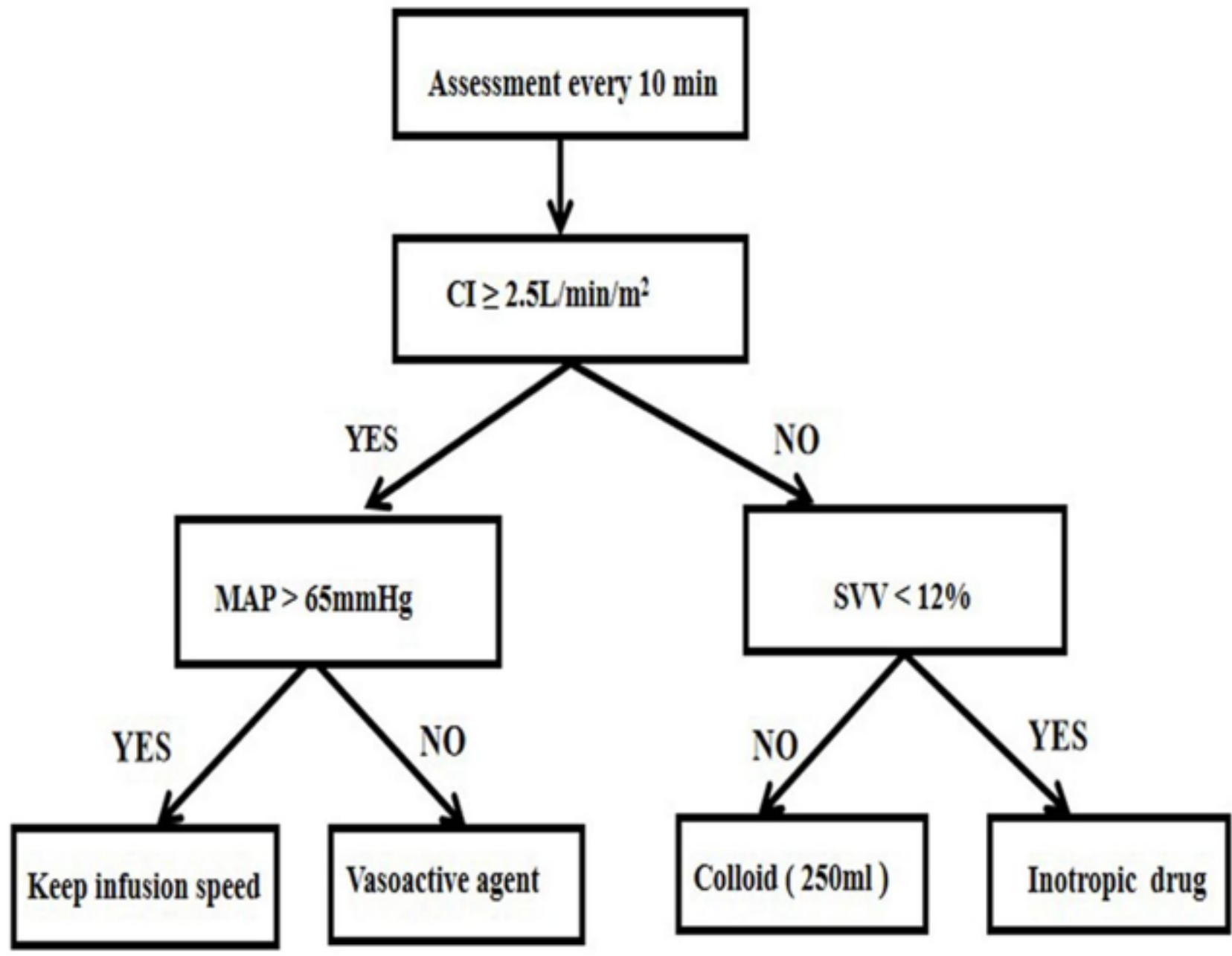

Figure 2

Flow chart of the GDFT 


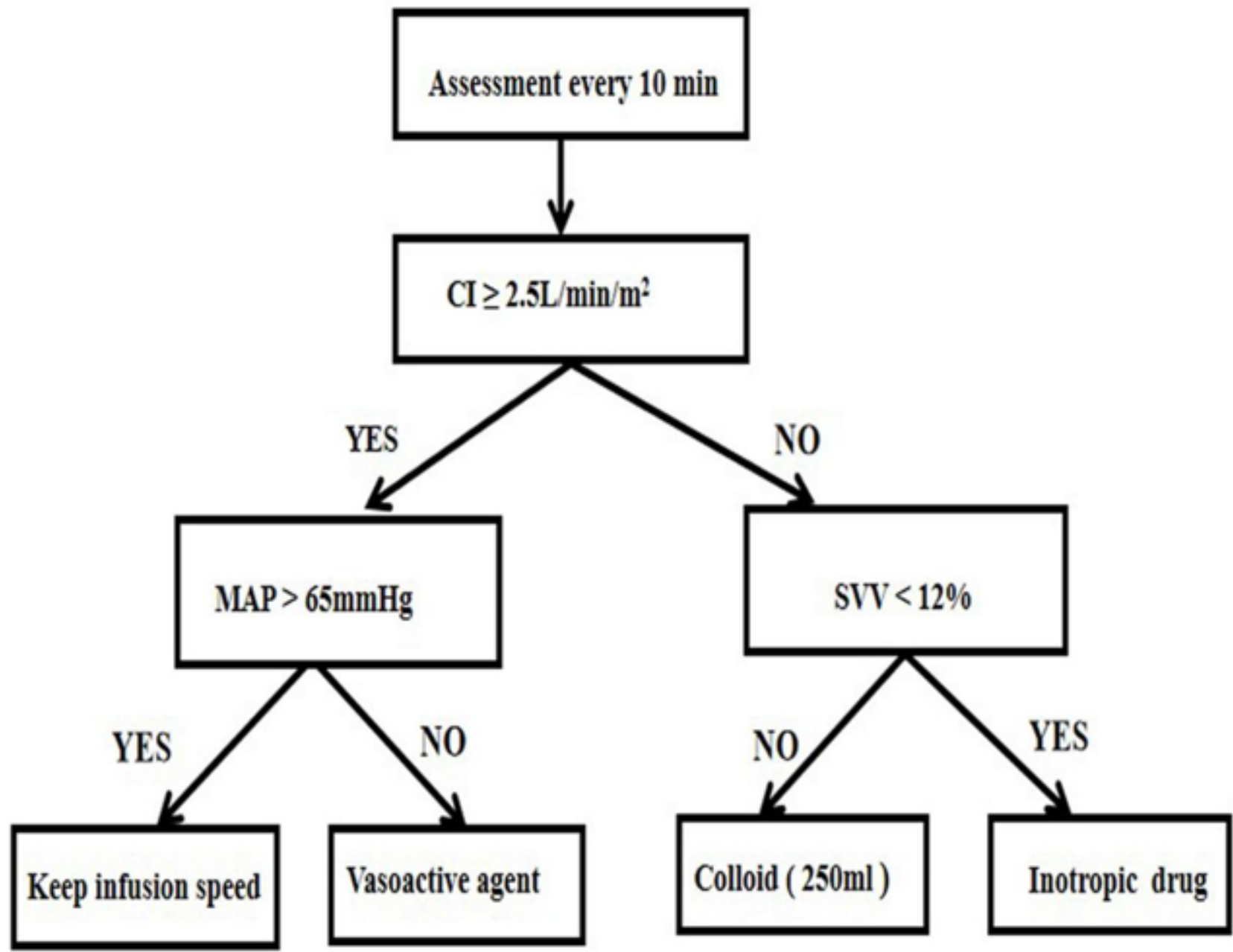

Figure 2

Flow chart of the GDFT

\section{Supplementary Files}

This is a list of supplementary files associated with this preprint. Click to download.

- Additionalfile1.docx

- Additionalfile1.docx

- CONSORT2010Checklist2.doc

- CONSORT2010Checklist2.doc 